\title{
Star accretion onto supermassive black holes in axisymmetric galactic nuclei
}

\author{
Shiyan Zhong ${ }^{1}$, Peter Berczik ${ }^{1,2,3}$ and Rainer Spurzem ${ }^{1,2,4}$ \\ ${ }^{1}$ National Astronomical Observatories of China, Chinese Academy of Sciences, 20A Datun \\ Rd., Chaoyang District, 100012, Beijing, China \\ ${ }^{2}$ Astronomisches Rechen-Institut, Zentrum für Astronomie, University of Heidelberg, \\ Mönchhofstrasse 12-14, 69120, Heidelberg, Germany \\ ${ }^{3}$ Main Astronomical Observatory, National Academy of Sciences of Ukraine, 27 Akademika \\ Zabolotnoho St., 03680, Kyiv, Ukraine \\ ${ }^{4}$ Kavli Institute for Astronomy and Astrophysics, Peking University, Beijing, China
}

\begin{abstract}
Tidal Disruption (TD) of stars by supermassive central black holes from dense rotating star clusters is modeled by high-accuracy direct $N$-body simulation. We study the time evolution of the stellar tidal disruption rate and the origin of tidally disrupted stars. Compared with that in spherical systems, we found a higher TD rate in axisymmetric systems. The enhancement can be explained by an enlarged loss-cone in phase space which is raised from the fact that total angular momentum $\mathbf{J}$ is not conserved. As in the case of spherical systems, the distribution of the last apocenter distance of tidally accreted stars peaks at the classical critical radius. However, the angular distribution of the origin of the accreted stars reveals bimodal features. We show that the bimodal structure can be explained by the presence of two families of regular orbits, namely short axis tube and saucer orbits.
\end{abstract}

Keywords. galaxies: kinematics and dynamics, galaxies: nuclei, methods: numerical, quasars: supermassive black holes, stars: kinematics and dynamics

\section{Introduction}

A large fraction of galaxies show evidence of supermassive black holes (henceforth $\mathrm{SMBH}$ ) residing in their center. They are typically embedded in nuclear star clusters (NSC). NSCs have size similar to galactic globular clusters, but they are much heavier and brighter (Böker et al. 2002; Böker et al. 2004). In massive galaxies NSCs may not be significant or even do not exist, however, the SMBHs are still surrounded by enormous number of stars. SMBH residing in these NSCs can tidally disrupt stars that come close to its tidal radius and eventually accrete the gaseous debris, which can light up the central SMBH for a period of time (Rees 1988; Evans \& Kochanek 1989). This kind of event is a useful tool to examine the relativistic physics near SMBH since the disruption occurs at a place very close to the BH's Schwarzschild radius. Also it can help us to investigate $\mathrm{SMBH}$ in non-active galactic center. Although tidal disruption of stars has been proposed for almost half a century, only until last decade do people realize the importance of such events, after the discovery of a dozens of tidal disruption candidates (Komossa 2002; Komossa \& Merritt 2008; Liu et al. 2014).

In order to compute the TD event rate, loss cone theory was developed (Frank \& Rees 1976; Lightman \& Shapiro 1977) and mostly assumed spherical symmetry of the stellar system. However, the host NSC of SMBH may not be spherical object as shown by both observation (Feldmeier et al. 2014; Schödel et al. 2014) and simulation (Antonini et al. 2012), which motivates us to study TD event in non-spherical systems. For this 


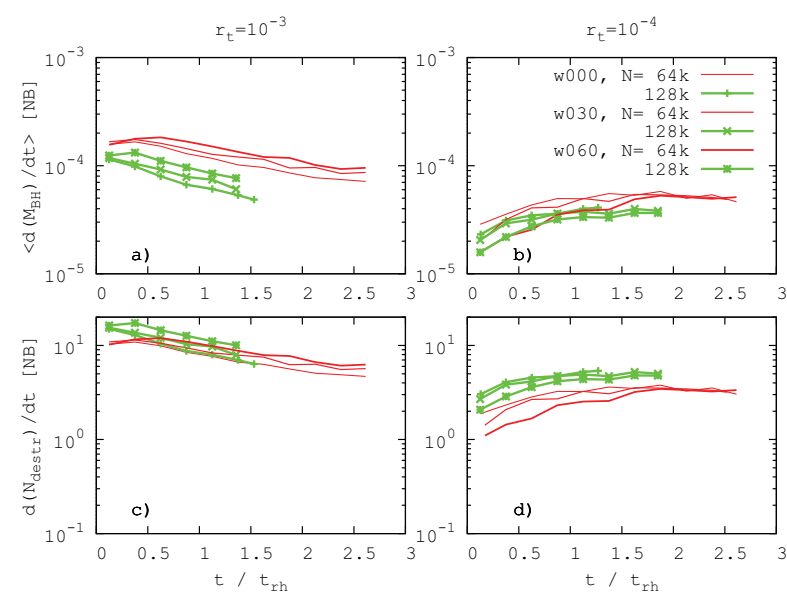

Figure 1. $x$ axis is time in unit of initial half-mass relaxation time. $y$ axis for panel a) and b) is the averaged mass accretion rate in given time range (i.e. $\left.1 / 4 t_{r h}\right) ; y$ axis for panel c) and d) is the number accretion rate. Panel a) and c) show the result for $r_{t}=10^{-3}$. Panel b) and d) show the result for $r_{t}=10^{-3}$. Line thickness indicate different rotating parameters.

purpose, we performed direct $N$-body simulations with model stellar clusters constructed by rotating King model (Ernst et al. 2007) and put a massive particle in the center which representing the SMBH. A fixed (and artificially enhanced) tidal radius is set for the SMBH $\left(r_{t}=10^{-3}, 10^{-4}\right.$ in model unit). We choose different rotating parameters $\left(\omega_{0}=0.0,0.3,0.6\right)$ to study the effect of rotation on TD. In the following sections we briefly report our results.

\section{Tidal disruption rate}

Fig. 1 summarizes the tidal disruption rate (TDR) results taken from simulations with different rotating parameters, particle numbers and tidal radius. TDR curves in left column represent large $r_{t}$ models. These models quickly enter empty loss cone regime. And faster rotation results in higher TDR. However, TDR curves in right column show an opposite trend: faster rotation results in lower TDR. These systems are still in full loss cone regime and $\mathrm{BH}$ has large Brownian motion which may cause this discrepancy. And TDR curves of different models converge at the late stage. After entering empty loss cone regime, their behaviors are consistent with that of large $r_{t}$ models. The results of $\omega_{0}=0.0$ models resemble those of Plummer model (Zhong et al. 2014).

\section{Loss cone shape in axisymmetric potential}

The enhancement of TDR could caused by faster relaxation process in rotating systems, or could be the consequence of an enlarged loss cone. In order to address this question, we perform test particle experiments to investigate the loss cone in axisymmetric potential.

In axisymmetric potential, the total angular momentum $J$ of a test particle is not conserved. As a result, star outside of the loss cone $\left(J>J_{l c} \approx \sqrt{2 G M_{\bullet} r_{t}}\right)$ has chance to drift into the loss cone and star with $J<J_{l c}$ can drift out. So the boundary of loss cone in $J$ dimension may be a few times larger than classical $J_{l c}$ (Magorrian \& Tremaine 1999). $J_{z}$ is still conserved and the condition $J_{z}<J_{l c}$ shall always be satisfied. At the time of disruption, $J<J_{l c}$ is required. We perform the experiment in phase space coordinated by energy $E$, angular momentum $J$ and its $z$ component $J_{z}$. Given a combination of 


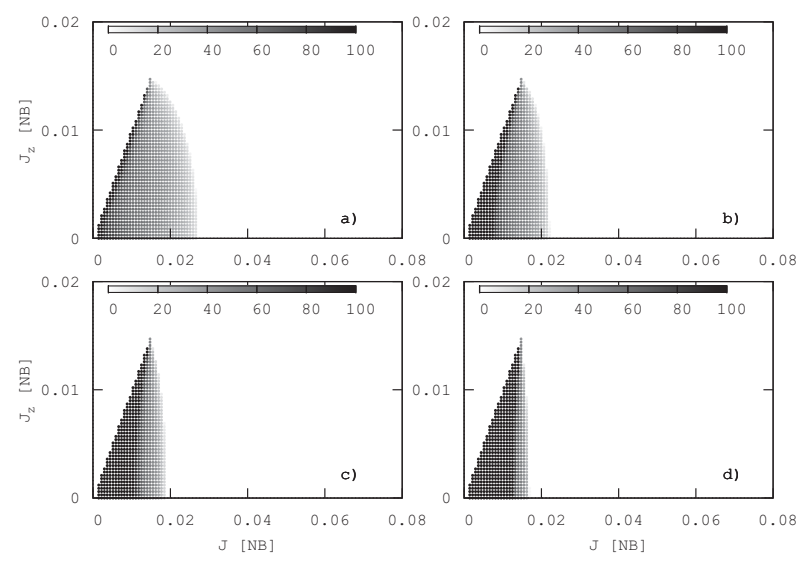

Figure 2. $x$ axis is module of angular momentum in $N$-body unit. $y$ axis is $z$ component of angular momentum. Panel a) correspond to $E=-1.3$, b) $E=-1.5$, c) $E=-1.7$, d) $E=-1.9$. Gray scale indicate the filling factor $P$ in percentage.

$\left(E, J, J_{z}\right)$, we put the test particle at its apocenter position and integrate its orbit for one orbital period in the potential generated with Self-Consistent Field (SCF, Hernquist \& Ostriker 1992) code, based on snapshots from the direct $N$-body simulation. If the particle reaches BH's tidal radius we mark it as loss cone star, otherwise it is out of loss cone. We also found that the fate of stars with same $\left(E, J, J_{z}\right)$ might be different, depending on the zenith angle $\theta$ of their apocenter. We incorporate these dependence into a parameter $P$ which means among all stars with same $\left(E, J, J_{z}\right)$ only a fraction $P$ of them is inside loss cone. The results are shown in Fig. 2.

The whole plane in each panel comprises 3 regions along $J$ axis: 1$)$ inner region where $P$ equals 1 , particles with these $\left(E, J, J_{z}\right)$ can definitely hit the $\mathrm{BH}$ within one dynamical time scale; 2 ) transition region where $P$ is non-zero but less than $1 ; 3$ ) outer region where $P=0$. Transition region shrinks from high energy to low energy case, which reflect the fact that variation of $J$ becomes smaller as energy decreases. Because in low energy case, test particles are close to the $\mathrm{BH}$, the potential is more spherical. While in high energy case, test particles can move to the outer region where the axisymmetric stellar potential dominate. We calculate the effective area of loss cone for different values of $E$ and find that in the energy range where most of the disrupted stars come from, the effective area of loss cone is larger comparing to spherical case, thus can account for the enhancement of TDR.

\section{Origin of disrupted stars}

Loss cone theory indicate that most of the disrupted stars should originate from the place around the critical radius, which is roughly the same as influence radius of the SMBH. We measure the last apocenter position for the disrupted stars and study their distribution in both $r$ and $\theta$ dimension. The $r$ distribution is similar to those obtained in spherical case (Fig. 3 left panel, also see Zhong et al. 2014). However, $\theta$ distribution show double peaks around the equatorial plane which is unexpected (Fig. 3, middle panel). After doing orbit classification for the disrupted stars, we find that this feature is caused by the different orbital types of the disrupted stars (Fig. 3, right panel).

Inside BH's influence radius $r_{h}$, there are two types of regular orbits, namely short axis tube (SAT) and saucer. The apocenter of SAT orbit can cross the equatorial plane and 

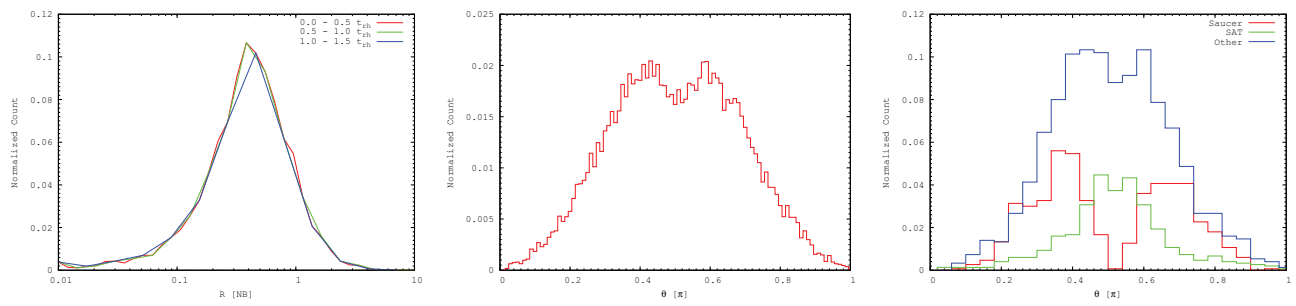

Figure 3. Normalized $r$ and $\theta$ distribution of last apocenter of disrupted stars.

minimum $J$ is acquired on the equatorial plane, so the last apocenter of disrupted stars moving on SAT orbit should concentrate to equatorial plane. The apocenter of saucer orbit avoid equatorial plane, the minimum $J$ is acquired at the region above or below it. Thus the saucer orbit is responsible for the double peaks. Outside $r_{h}$, star orbits are mostly chaotic, thus $\theta$ distribution do not have the double-peak feature.

\section{Conclusion}

In our $N$-body simulations we find that $\mathrm{BHs}$ in axisymmetric system exhibit higher TD rate than spherical system. This enhancement is caused by an enlarged loss cone in phase space. Most of the disrupted stars are coming from place far from the central $\mathrm{BH}$. The distribution of their angular position show double-peak feature, which can be explained by the different orbit types of the disrupted stars.

\section{References}

Antonini, F., Capuzzo-Dolcetta, R., Mastrobuono-Battisti, A. \& Merritt, D. 2012 ApJ 750, 111 Böker, T., Laine, S., van der Marel, R. P., Sarzi, M., Rix, H.-W., Ho, L. C., \& Shields, J. C. 2002, AJ, 123, 1389

Böker, T., Sarzi, M., McLaughlin, D. E., van der Marel, R. P., Rix, H.-W., Ho, L. C., \& Shields, J. C. $2004, A J, 127,105$

Ernst, A., Glaschke, P., Fiestas, J., Just, A. \& Spurzem, R. 2007 MNRAS 377, 465

Evans, C. R. \& Kochanek, C. S. 1989, ApJL, 346, L13

Feldmeier, A., Neumayer, N., Seth, A., Schödel, R., Lützgendorf, N., de Zeeuw, P. T., KisslerPatig, M., Nishiyama, S. \& Walcher, C. J. 2014 A\&A 570, A2

Frank, J. \& Rees, M. J. 1976 MNRAS 176, 633

Hernquist, L. \& Ostriker, J. P. 1992 ApJ 386, 375

Komossa, S. 2002 Reviews in Modern Astronomy, 15, 27

Komossa, S. \& Merritt, D. 2008 ApJL 683, L21

Lightman, A. P. \& Shapiro, S. L. 1976 ApJ 211, 244

Liu, F. K., Li, S. \& Komossa, S. 2014 ApJ 786, 103

Magorrian, J. \& Tremaine, S. 1999 MNRAS 309, 447

Rees, M. J. 1988, Nature, 333, 523

Schödel, R., Feldmeier, A., Kunneriath, D., Stolovy, S., Neumayer, N., Amaro-Seoane, P. \& Nishiyama, S. 2014 A $\dot{E} A$ 566, A47

Zhong, S., Berczik, P. \& Spurzem, R. 2014 ApJ 792, 137 